\title{
Experience of Clinical Management for Pregnant Women and Newborns with Novel Coronavirus Pneumonia in Tongji Hospital, China
}

\author{
Shao-shuai WANG ${ }^{1 \dagger}$, Xuan ZHOU ${ }^{1 \dagger}$, Xing-guang LIN ${ }^{1}$, Yan-yan LIU ${ }^{1}$, Jian-li WU ${ }^{1}$, Lali Mwamaka Sharifu ${ }^{1}$, Xiao-lin HU², \\ Zhi-hui RONG ${ }^{2}$, Wei LIU², Xiao-ping LUO ${ }^{2}$, Zhuo CHEN ${ }^{3}$, Wan-jiang ZENG ${ }^{1}$, Su-hua CHEN ${ }^{1}$, Ding MA ${ }^{1}$, Ling CHEN ${ }^{2 \#}$, Ling \\ FENG $^{1 \#}$ \\ ${ }^{1}$ Department of Obstetrics and Gynecology, Tongji Hospital, Tongji Medical College, Huazhong University of Science and \\ Technology, Wuhan 430030, China \\ ${ }^{2}$ Department of Pediatrics, Tongji Hospital, Tongji Medical College, Huazhong University of Science and Technology, Wuhan \\ 430030 China \\ ${ }^{3}$ Department of Traditional Chinese Medicine, Tongji Hospital, Tongji Medical College, Huazhong University of Science and \\ Technology, Wuhan 430030, China
}

(C) Huazhong University of Science and Technology 2020

\begin{abstract}
Summary: Based on the New Diagnosis and Treatment Scheme for Novel Coronavirus Infected Pneumonia (Trial Edition 5), combined with our current clinical treatment experience, we recently proposed a revision of the first edition of "Guidance for maternal and fetal management during pneumonia epidemics of novel coronavirus infection in the Wuhan Tongji Hospital”. This article focused on the issues of greatest concern of pregnant women including severe acute respiratory syndrome coronavirus 2 (SARS-CoV-2) infection diagnostic criteria, inspection precautions, drug treatment options, indications and methods of termination of pregnancy, postpartum fever, breastfeeding considerations, mode of mother-to-child transmission, neonatal isolation and advice on neonatal nursing, to provide valuable experience for better management of SARS-CoV-2 infection in pregnant women and newborns.
\end{abstract}

Key words: COVID-19; pneumonia; pregnant women; newborn; disease management

\section{INTRODUCTION}

Since December 2019, Wuhan-Hubei province, China has successively found several cases of patients with novel coronavirus pneumonia (NCP). With the spread of the severe acute respiratory syndrome coronavirus 2 (SARS-CoV-2), such cases have been found in other regions of China and abroad, and some areas have found cases with no travel history in Wuhan. This disease has been incorporated into the Class B infectious diseases stipulated in the Law of the People's Republic of China on the Prevention and Control of Infectious Diseases and measures for the prevention and management of Class A infectious diseases have been adopted.

On February 5, 2020, the National Health Commission of the People's Republic of China issued the New Diagnosis and Treatment Scheme for Novel Coronavirus Infected Pneumonia (Trial Edition

Shao-shuai WANG, E-mail: colombo2008@sina.com;

Xuan ZHOU, E-mail: zhouxuantjh@163.com

${ }^{\dagger}$ The authors contributed equally to this work.

\#Corresponding authors, Ling FENG, E-mail: fltj007@163.

com; Ling CHEN, E-mail: 790356760@qq.com.
5) ${ }^{[1]}$ (hereinafter referred to as the "Fifth Edition of Diagnosis and Treatment Scheme"), which pointed out that asymptomatic patients could also spread the virus. Most patients had fever, fatigue, dry cough as the mainly manifested symptoms and a few patients had nasal congestion, runny nose, diarrhea and other symptoms, while several cases had no obvious fever. The total number of leukocytes in peripheral blood in the early stage of disease was normal or reduced, the lymphocyte count was generally decreased, and chest CT presented NCP's imaging features ${ }^{[2]}$.

Special attention should be paid to the different requirements of Hubei Province and other provinces in terms of the epidemic's situation, the diagnostic criteria of the disease, and the detection, reporting and exclusion of cases. In addition to suspected and confirmed cases, clinically diagnosed cases have been added in Hubei Province. As long as the suspected cases have NCP's imaging features, they would be treated as clinically diagnosed cases and handled according to the severity of clinical classification. Once suspected cases and clinically diagnosed cases are found in Hubei Province, they would be immediately quarantined, treated, and specimens taken for pathogenic testing as soon as possible. 
Pregnant women are in a state of special immune tolerance and are susceptible to the virus. There has been many confirmed cases of infection in pregnant women in China. We have recently reported a case of neonatal infection with throat swab virus positive at 36 $\mathrm{h}$ after birth ${ }^{[3]}$. Therefore, the diagnosis and treatment plan for suspected or confirmed cases in pregnant women must take into account the situation of both mothers and babies. On January 28, we published "Guidance for maternal and fetal management during pneumonia epidemics of novel coronavirus infection in the Wuhan Tongji Hospital (First edition)" ${ }^{\text {"[4]. Based }}$ on the clinical characteristics, diagnosis and treatment progress of the recently discovered diseases, we offered an updated clinical management for pregnant women and newborns with NCP.

\section{MATERNAL MANAGEMENT}

\subsection{Diagnostic Criteria}

According to the "Fifth Edition of Diagnosis and Treatment Scheme", the diagnostic criteria of Hubei Province and other provinces are different. The cases are still divided into suspected and confirmed cases in provinces outside Hubei. The specific standards have been presented in the "Fifth Edition of Diagnosis and Treatment Scheme". In Hubei Province, in addition to suspected and confirmed cases, clinically diagnosed cases have been added. As long as the suspected case has imaging characteristics of NCP, it will be judged as a clinically diagnosed case, and its clinical classification and treatment principles are consistent with those of the confirmed cases. It emphasizes the importance of clinical diagnosis of imaging, which is conducive to early isolation and early treatment. Details are as follows:

(1) Suspected cases should satisfy any one or none of the items of epidemiological history, and simultaneously meet clinical manifestations at the same time. Comprehensive analysis of the following epidemiological history and clinical manifestations should be applied.

Epidemiological history: (1) Travel history or residence history in Wuhan, or surrounding areas near Wuhan, or other communities with case reports within 14 days before the onset of illness; (2) Contact history with patients infected with SARS-CoV-2 (positive nucleic acid test) within 14 days before the onset of illness; (3) Contact history with patients who had a fever or patients with respiratory symptoms from Wuhan or surrounding areas near Wuhan or from communities with case reports within 14 days before the onset of illness; (4) Clustering occurrence.

Clinical manifestations: (1) Fever or respiratory symptoms; (2) Reduction in the total number of leukocytes or normal in the early stage of diseases, or the lymphocyte count is reduced. Note that the blood cells count may not have typical changes during the early stages of the infection in pregnant women.

(2) Clinically diagnosed cases refer to suspected cases that have imaging characteristics of NCP.

(3) Confirmed cases signify clinically diagnosed or suspected cases with one of the following pathogenic evidence: (1) Real-time quantitative PCR of respiratory specimens or blood specimens is positive for the detection of SARS-CoV-2 nucleic acids; (2)Viruses in respiratory specimens or blood specimens for genetically sequenced are highly homologous to known SARS-CoV-2.

\subsection{Institution and Procedure of Hospitalization}

Suspected cases, particularly cases with fever in pregnant women should be firstly pre-diagnosed, temperature checked and directed by a clinician to designated fever clinics. Almost all local health commissions have successively set up designated hospitals for patients with corona virus disease 2019 (COVID-19) including some designated hospitals specialized in pregnant women.

Suspected or confirmed cases outside Hubei Province should be quarantined immediately and on the promise of ensuring the safety of transfer, they should be transferred to the designated hospital that can treat pregnant women as soon as possible. Suspected and clinically diagnosed cases in Hubei Province must be immediately quarantined and treated in a single room, and confirmed cases can be treated in the same ward.

\subsection{Criteria for Recovery}

If the temperature reverts to normal for more than 3 days, the respiratory symptoms improves significantly, the lung imaging shows obvious absorption of inflammation, and the nucleic acid test for respiratory pathogens shows negative for two consecutive times (sampling interval at least 1 day), the patient could discharge from the hospital or be transferred to the appropriate department for treatment according to other illnesses.

\subsection{Inspection Precautions}

Even if eight routine tests for respiratory virus infection are positive, it still cannot exclude SARS$\mathrm{CoV}-2$ infection. According to existing clinical cases, patients with NCP may be infected with pathogens such as influenza A virus, influenza B virus, and mycoplasma at the same time. Therefore, for suspected cases, clinically diagnosed cases and those with a history of close contact, it is recommended that SARS$\mathrm{CoV}-2$ pathogenic tests are performed in time even if the eight routine tests for respiratory virus infection are positive. Moreover, it was clinically found that the positive rate of nucleic acid detection in patients with NCP could not reach $100 \%$. If the test result is negative, the test is performed again if necessary.

Whether the radiological examination causes 
abnormal fetal development depends on the gestational week at that time and the fetal radiation dose of the radiological examination technique. Theoretically, the fetal radiation dose of chest CT does not reach the teratogenic threshold, so it is relatively safe. It is recommended that pregnant women take a chest CT examination after informed consent, and take abdominal protection measures for the sake of safety.

\subsection{Drug Treatment Options}

The use of alpha-interferon in the early stage of pregnancy has the risk of affecting fetal growth and development, hence pregnant women should be fully informed. Lopinavir/ritonavir have been listed as the preferred drug regimen for HIV during pregnancy ${ }^{[5]}$. Based on animal studies and limited human reports, the drug does not appear to increase the risk of adverse pregnancy outcomes. Medical personnel should bring in pregnant women and their families on the benefits for pregnant women and the potential risks for the fetus, and choose the medicine when the potential benefits outweigh the potential risks to the fetus. Obvious embryonic lethality and teratogenicity were observed in ribavirin in animal experiments, thus, it was contraindicated during pregnancy.

Suspected cases as well as mild and ordinary cases in pregnant women could take traditional Chinese medicines for a week. Patients with fatigue and fever could take Jinye Baidu granules, 1 bag at a time, 3 times a day or take Lianhua Qingwen capsule, 4 capsules at a time, 3 times a day. For those with asthenia and gastrointestinal discomfort, Huoxiang Zhengqi (concentrated honey pill or oral liquid) can be selected, 8 capsules or 1 stick at a time, 3 times a day. Patients with diarrhea can choose berberine $0.3 \mathrm{~g}$ once, 2-3 times a day. It is advocated to take a cure according to the prescription of gynecologists of traditional Chinese medicine. There is no safety data currently in pregnant women taking the above mentioned traditional Chinese medicines, but the antiviral efficacy has been recognized. Pregnant women should be informed to weigh the advantages and disadvantages before choosing to use them. Because the pregnant women infected with SARS-CoV-2 are not recommended to breastfeed, taking traditional Chinese medicines might be safe.

\subsection{Antenatal Examination}

During the epidemic, it is suggested that pregnant women perform prenatal inspection and assessment in accordance with the guidelines for health care under the promise of personal protection during pregnancy. It is necessary to pay close attention to fetal movement changes during the third trimester. Those with complications should increase the frequency of the prenatal inspections. Suspected cases, clinically diagnosed cases and confirmed cases in pregnant women should undergo obstetric examinations at designated hospitals set by the local health commission that can treat pregnant women. Electronic fetal heart rate monitoring and ultrasound examinations should be used to assess the condition of the fetus based on the gestation week. Other pregnant women are advised to complete the obstetric examination at a medical institution that does not have fever clinics. If it is possible, it is recommended that medical institutions that carry out maternal health care during the NCP epidemic establish green channels for pregnant women ${ }^{[6]}$. In order to reduce cross-infection, medical institutions that have perinatal health care should take advantage of the Internet Plus to fulfill online consultation and guidance for pregnant women.

\subsection{Indications and Methods of Terminating Pregnancy}

Before terminating pregnancy during the epidemic, a comprehensive analysis of the severity of the disease, the gestational week and the fetal condition should be taken into account ${ }^{[7]}$. Indications for termination of pregnancy include: (1) Obstetric indications: Judging according to the specific situation and indications for termination of pregnancy. (2) Severe and critical cases: Continuing pregnancy may endanger the safety of the mother and fetus. On the basis of informed consent, pregnancy should be terminated.

Severe cases refer to respiratory distress (respiratory rate $\geq 30$ beats $/ \mathrm{min}$ ); or oxygen saturation $\leq 93 \%$ at rest; or arterial blood oxygen pressure $\left(\mathrm{PaO}_{2}\right) /$ oxygen concentration $\left(\mathrm{FiO}_{2}\right) \leq 300 \mathrm{mmHg}$. Critically ill patients refer to cases with respiratory failure and requirement for mechanical ventilation; or shock; or complications of other organ failure requiring ICU monitoring and treatment.

The principles of how to terminate pregnancy are as follows:

(1) Indications for vaginal delivery: mild illness, good cervical conditions, already in labor and no contraindications to vaginal delivery.

(2) Indications for termination by surgery: severe and critical cases.

The sites of termination of pregnancy for suspected or confirmed cases are dedicated isolation rooms or operating rooms for respiratory infectious diseases in the fever ward. A multidisciplinary joint diagnosis and treatment team should be set up, composed of medical staff in various specialties such as obstetrics, neonatal, infection, respiratory and anesthesia. Operating room should be established and special surgery and anesthetic items must be prepared ${ }^{[6]}$. It is important to note that one case of neonatal infection with pharyngeal swab virus positive in a $36-\mathrm{h}$ baby has been reported recently ${ }^{[3]}$. The existence of mother-to-child transmission of SARS-CoV-2 has not been confirmed, although it was reported on the Lancet that there was no vertical transmission of SARS-CoV-2 ${ }^{[8]}$. It is necessary 
to pay attention to the protection for newborns during delivery and clean the newborns as soon as possible.

\subsection{Postpartum Fever}

After the pregnant giving birth, attention should be paid to maternal fever. Postpartum fever may occur due to the following physical characteristics: decreased immunity originated from fatigue and blood loss in childbirth, anatomy of female genitalia, sweating during puerperium, and postpartum lactation. Once postpartum fever occurs, differential diagnosis to exclude breast swelling, mastitis, urinary tract infections, common colds, and reproductive tract infections must be focused on. Those with SARSCoV-2 infected pneumonia-related symptoms should perform blood routine, respiratory virus testing and chest $\mathrm{CT}$ examinations in a timely manner. If chest CT shows imaging characteristics of NCP, the patient should complete SARS-CoV-2 nucleic acid tests immediately. In the fever clinics, obstetricians and gynecologists should attend to rule out obstetric fever if necessary.

\subsection{Breastfeeding Considerations}

Close attention must be paid to the hygiene of hands and breasts during breastfeeding. Suspected cases, uncured clinically diagnosed cases and uncured confirmed cases are not recommended to breastfeed. Lopinavir/ritonavir can be secreted with the milk of rats but it is uncertain whether the breast milk in human contains ingredients of the drug. Therefore, breastfeeding is not suggested while taking this medicine. During the suspension of breastfeeding, it is advised that the mother empties her breasts regularly.

\section{FETAL MANAGEMENT}

\subsection{Diagnostic Criteria}

Two conditions need to be met at the same time for the diagnosis of newborns: (1) Meet the diagnostic criteria of Fifth Edition of Diagnosis and Treatment Scheme; (2) SARS-CoV-2 nucleic acid test is positive regardless of whether the newborn has developed symptoms or not.

\subsection{Mode of Mother-to-child Transmission}

It has been reported that a case of SARS-CoV-2 infection with positive nucleic acid of pharyngeal swab virus occurred in a baby only $36 \mathrm{~h}$ after birth ${ }^{[3]}$, and the mother was a confirmed case of SARS-CoV-2 infection. Therefore, the existence of mother-to-child transmission of SARS-CoV-2 remains to be verified.

The possible ways of SARS-CoV-2 infection are: vertical transmission from mother to fetus, close contact transmission, droplet transmission (family members, family visitors) and hospital-acquired infections.

Newborns who meet any one of the followings need to be examined: (1) Pregnant women were confirmed or suspected to SARS-CoV-2 infection;
(2)Pregnant women were in close contact with family members who were confirmed or highly suspected of being infected; (3) In the process of caring for newborns, family members were confirmed or highly suspected to infection.

If the above situations happen, the newborn should be isolated and observed immediately as soon as possible, and nucleic acid tests should be carried out, too. In addition, breastfeeding should not be performed if the mother was infected by SARS-CoV- ${ }^{[9]}$.

\subsection{Neonatal Isolation}

Newborns who have been confirmed with SARSCoV-2 infection must be transferred to an isolated observation ward for at least 14 days. If the delivery institution does not have the conditions of a neonatal isolation ward, the newborn must be transferred to designated medical institutions that can isolate and treat for them. Newborns suspected of having SARSCoV-2 infection should also be transferred to an isolation ward for observation or treatment until the recovery standard is met. Only when the mother is negative of two consecutive SARS-CoV-2 nucleic acid tests (interval of 1 day) and under informed consent, the mother and child can be in the same room ${ }^{[10]}$.

\subsection{Advice on Neonatal Nursing}

During the COVID-19 epidemic, if the newborn has no history of contact and no special discomfort, such as fever, there is no need for routine investigation but normal newborn care is required. Preventive and control measures should be taken during home care: single rooms with appropriate temperature and humidity, minimizing caregivers, regular window ventilation, caregivers washing hands and face often, disinfecting the daily supplies of newborns, etc. It is recommended to use $75 \%$ medical alcohol and chlorine-containing disinfection water to wipe the floor and furniture. Heat-resistant bottles and pacifiers should be disinfected at high temperature ${ }^{[11,12]}$.

If mothers have been diagnosed or suspected to have SARS-CoV-2 infection and newborns have not yet completed disease screening and hearing testing, they can go to the local maternity and child health care institutions for additional newborn disease screening and hearing testing after the end of the epidemic. If vitamin $\mathrm{K} 1$ is not added to newborns at birth, supplementation is also required.

Pregnant women are in a state of special immune tolerance. The mucosa of upper respiratory tract (nose, pharynx, trachea) is thickened, slightly congested and edematous. In addition, immunity of newborns is extremely poor. Both mothers and newborns are prone to suffer from upper respiratory tract infection. Meanwhile, because of being susceptible to SARS$\mathrm{CoV}-2$, pregnant women are more likely to develope complications or even progress to severe cases after being infected with SARS-CoV-2 ${ }^{[13]}$, prevention and 
treatment become especially significant. This article was formulated based on the currently available case data and our experience of diagnosis and treatment. According to the development of the epidemic and the accumulation of experience in diagnosis and treatment, the relevant content remains to be gradually improved.

\section{Conflict of Interest Statement}

None.

\section{REFERENCES}

1 National Health Commission of the People's Republic of China. New diagnosis and treatment scheme for novel coronavirus infected pneumonia (Trial edition 5) (Chinese) [R/OL]. [2020-02-05].

2 Medical Expert Group, Tongji Hospital, Tongji Medical College, Huazhong University of Science and Technology. Quick guide for the diagnosis and treatment of pneumonia infected by novel coronavirus (Third edition) (Chinese)[EB/OL].[2020-01-28].

3 Wang SS, Guo LL, Chen L, et al. A case report of neonatal COVID-19 infection in China [J/OL]. Clinical infectious diseases: an official publication of the Infectious Diseases Society of America [2020-3-12].

4 Department of Obstetrics and Gynecology, Neonatal Department, Tongji Hospital, Tongji Medical College, Huazhong University of Science and Technology. Guidance for maternal and fetal management during pneumonia epidemics of novel coronavirus infection in the Wuhan Tongji Hospital (First edition) (Chinese) [EB/OL].[2020-01-28].

5 AIDS and Hepatitis C Professional Group, Society of Infectious Diseases, Chinese Medical Association, et al. Chinese guidelines for diagnosis and treatment of HIV/ AIDS (2018) (Chinese). Infectious Dis Information, 2018,06:481-499

6 Obstetric Disease Quality Control Center in Liaoning Province, Critical Maternal Rescue Center in Liaoning Province, Maternal and Fetal Medical Center in Liaoning Province. Guidance on maternal management during the epidemics of novel coronavirus in Liaoning province (First edition) (Chinese) [J/OL]. Chin J Pract Gynecol Obstet [2020-01-26].
7 World Health Organization. Clinical management of severe acute respiratory infection when novel coronavirus (2019-nCoV) infection is suspected: Interim guidance [EB/OL]. [2020-02-04].

8 Chen HJ, Guo JJ, Wang C, et al. Clinical characteristics and intrauterine vertical transmission potential of COVID-19 infection in nine pregnant women: a retrospective review of medical records $[\mathrm{J} / \mathrm{OL}]$. Lancet [2020-02-12].

9 Department of Obstetrics and Gynecology, Neonatal Department, Union Hospital, Tongji Medical College, Huazhong University of Science and Technology. Recommendations for management of pregnancy complicated with novel coronavirus infection (Second edition) (Chinese) [EB/OL]. [2020-01-28].

10 Neonatal Physician Branch of Chinese Physician Association, Neonatal Health Professional Committee of China Maternal and Child Health Association, Perinatal Medical Branch of Chinese Medical Association, Editorial Board of Chinese Journal of Perinatal Medicine. Expert recommendations for the prevention and control of 2019 novel coronavirus infection in neonatal department. Chin J Perinatal Med, 2020,23(02):80-84

11 World Health Organization. Home care for patients with suspected novel coronavirus (2019-nCoV) infection presenting with mild symptoms and management of contacts: Interim guidance [EB/OL]. [2020-02-04].

12 Centers for Disease Control and Prevention. Prevention of the transmission of novel coronavirus (2019-nCoV) to families and communities in 2019: Interim guidance [R/OL].

13 Maternal and Fetal Physician Professional Committee of the Chinese Physician Association of Obstetricians and Gynecologists, Obstetrics Group of the Chinese Medical Association of Obstetrics and Gynecology Branch, Chinese Medical Association Perinatal Medicine Branch, Editorial Board of Chinese Journal of Perinatal Medicine. Expert suggestion on novel coronavirus infection during pregnancy and postpartum period (Chinese). Chin J Perinatal Med, 2020,23(02):7379

(Received Feb. 12, 2020; revised Mar. 14, 2020) 